\title{
Alterstice
}

Revue internationale de la recherche interculturelle

International Journal of Intercultural Research

Revista International de la Investigacion Intercultural

\section{Droits humains et témoignages : l'épreuve de la culture}

\section{Francine Saillant}

Volume 1, numéro 2, 2011

Droits humains et témoignages : l'épreuve de la culture

URI : https://id.erudit.org/iderudit/1077598ar

DOI : https://doi.org/10.7202/1077598ar

Aller au sommaire du numéro

\section{Éditeur(s)}

Alterstice

ISSN

1923-919X (numérique)

Découvrir la revue

Citer ce document

Saillant, F. (2011). Droits humains et témoignages : l'épreuve de la culture. Alterstice, 1(2), 3-8. https://doi.org/10.7202/1077598ar

\section{Résumé de l'article}

Les violences contemporaines qui impliquent dans nombre de cas des exactions des droits les plus fondamentaux sont chaque jour exposées à nos yeux, ne serait-ce que par leur hypermédiatisation en tous genres. Les droits humains sont pour leur part devenus plus que jamais un langage transnational qui permet la traduction multiforme et culturellement située de ces exactions. Le témoignage, utilisé de diverses façons comme forme de communication des exactions par rapport aux droits, mérite une attention particulière. Le témoignage, dont l'usage est connu dans le domaine du droit en tant que moyen de faire apparaître la vérité, prend potentiellement dans l'espace public d'autres significations. Il est plus que jamais l'une des expressions des altérités, en même temps qu'un moyen, pour diverses catégories d'étrangers et de personnes et collectivités minorisées et discriminées, d'arriver par la voix ou l'image à se faire entendre et voir. Se faire entendre, c'est-à-dire que la voix puisse avoir écho et réponse, mais aussi que cette voix soit recevable et reconnue. Se faire voir c'est-à-dire ouvrir un espace pour des images qui font apparaître les sujets, leurs droits lésés, leurs revendications, leurs actions, leurs victoires. Ce numéro propose une réflexion approfondie sur cette question : d'abord en exposant une série de cas articulant droits et témoignages; ensuite en cernant, leur variété aidant, les multiples médiations qui font que ces voix seront oui ou non entendues et reconnues, ces images plus ou moins vues et reçues. Les cultures d'origine et de réception, qu'elles soient liées à l'ethnicité, aux institutions, au statut, à l'âge ou aux régimes politiques, jouent comme on le verra, un rôle fondamental. 


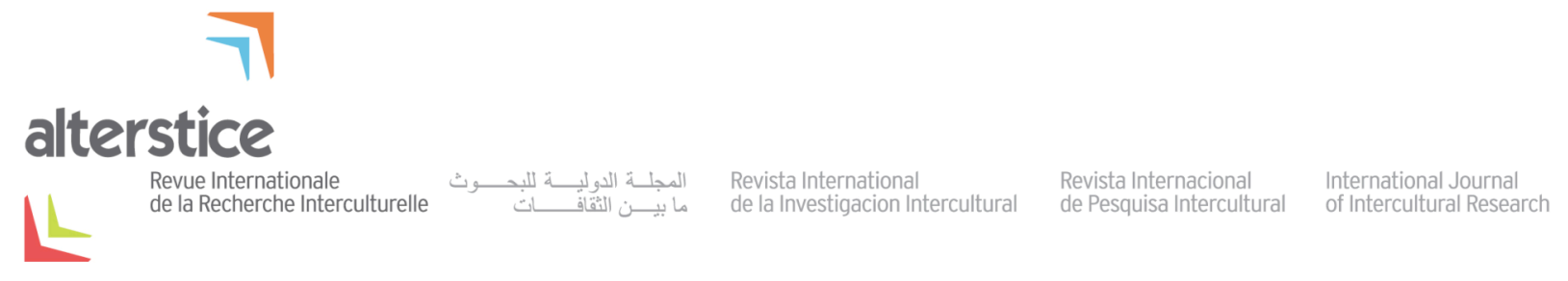

INTRODUCTION THÉMATIQUE

\section{Droits humains et témoignages : l'épreuve de la culture}

Francine Saillant ${ }^{1}$

\section{Résumé}

Les violences contemporaines qui impliquent dans nombre de cas des exactions des droits les plus fondamentaux sont chaque jour exposées à nos yeux, ne serait-ce que par leur hypermédiatisation en tous genres. Les droits humains sont pour leur part devenus plus que jamais un langage transnational qui permet la traduction multiforme et culturellement située de ces exactions. Le témoignage, utilisé de diverses façons comme forme de communication des exactions par rapport aux droits, mérite une attention particulière. Le témoignage, dont l'usage est connu dans le domaine du droit en tant que moyen de faire apparaître la vérité, prend potentiellement dans l'espace public d'autres significations. II est plus que jamais l'une des expressions des altérités, en même temps qu'un moyen, pour diverses catégories d'étrangers et de personnes et collectivités minorisées et discriminées, d'arriver par la voix ou l'image à se faire entendre et voir. Se faire entendre, c'est-à-dire que la voix puisse avoir écho et réponse, mais aussi que cette voix soit recevable et reconnue. Se faire voir c'est-à-dire ouvrir un espace pour des images qui font apparaître les sujets, leurs droits lésés, leurs revendications, leurs actions, leurs victoires. Ce numéro propose une réflexion approfondie sur cette question : d'abord en exposant une série de cas articulant droits et témoignages; ensuite en cernant, leur variété aidant, les multiples médiations qui font que ces voix seront oui ou non entendues et reconnues, ces images plus ou moins vues et reçues. Les cultures d'origine et de réception, qu'elles soient liées à l'ethnicité, aux institutions, au statut, à l'âge ou aux régimes politiques, jouent comme on le verra, un rôle fondamental.

\section{Rattachement de l'auteure}

${ }^{1}$ Département d'anthropologie et Centre interuniversitaire d'Étude sur les Lettres, les Arts et les Traditions (CÉLAT), Université Laval, Québec, Canada

\section{Correspondance}

francine.saillant@ant.ulaval.ca

\section{Mots clés}

témoignages; droits humains; migrants; réfugiés; image

\section{Pour citer cet article :}

Saillant, F. (2011). Droits humains et témoignages : l'épreuve de la culture. Alterstice, 1(2), 3-8. 
Les violences contemporaines, qui impliquent dans nombre de cas des exactions des droits les plus fondamentaux, sont chaque jour exposées à nos yeux, ne serait-ce que par leur hypermédiatisation en tous genres. Les droits humains ${ }^{1}$ sont pour leur part devenus plus que jamais un langage transnational qui permet la traduction multiforme et culturellement située de ces exactions (Engle, 2006). Le témoignage, utilisé de diverses façons comme forme de communication des exactions par rapport aux droits, mérite une attention particulière. Le témoignage, dont l'usage est connu dans le domaine du droit en tant que moyen de faire apparaître la vérité, prend potentiellement dans l'espace public d'autres significations. Il est plus que jamais l'une des expressions des altérités, en même temps qu'un moyen, pour diverses catégories d'étrangers et de personnes et collectivités minorisées et discriminées, d'arriver par la voix ou l'image à se faire entendre et voir. Se faire entendre, c'est-à-dire que la voix puisse avoir écho et réponse, mais aussi que cette voix soit recevable et reconnue. Se faire voir, c'est-àdire ouvrir un espace pour des images qui font apparaitre les sujets, leurs droits lésés, leurs revendications, leurs actions, leurs victoires. Ce numéro propose une réflexion approfondie sur cette question : d'abord en exposant une série de cas articulant droits et témoignages; ensuite en cernant, leur variété aidant, les multiples médiations qui font que ces voix seront oui ou non entendues et reconnues, ces images plus ou moins vues et reçues. Les cultures d'origine et de réception, qu'elles soient liées à l'ethnicité, aux institutions, au statut, à l'âge ou aux régimes politiques, jouent, comme on le verra, un rôle fondamental.

\section{Droits humains et témoignages}

Depuis une quinzaine d'années maintenant, les droits humains comme champ d'étude ont pris un essor fulgurant, en particulier en anthropologie. L'anthropologie, dont le rôle fut longtemps mineur dans l'étude des pratiques et des discours entourant les droits, parce que la discipline refusait la possibilité du langage universel qu'ils représentaient de même que leur construction occidentale, a quelque peu transformé son regard, en remettant en cause la perspective relativiste qui l'a traditionnellement marquée. Les possibilités ouvertes par l'anthropologie des droits telle qu'elle s'élabore actuellement, en abordant ces derniers en tant que pratiques sociales, viennent encadrer, en amont, l'étude du témoignage et de ses usages, entre autres à des fins de droits.

En effet, au cours des dernières années, les publications rapprochées d'articles importants dans des des revues comme Current Anthropology (2006) et American Anthropologist (2006) (voir Goodale 2006a et 2006b) sur la question des droits humains, puis d'ouvrages aussi fondamentaux que ceux de Sheper-Hugues et Bourgeois (2004), de Goodale et Merry (2008), de Goodale (2009), mais aussi en Europe de Hastrup (2001a et 2001b) et RudeAntoine et Chrétien-Vernicois (2009), sont des indications de l'importance croissante qu'a pris le champ au sein de la discipline, un champ, il va sans dire, qui se situe dans un carrefour interdisciplinaire faisant appel au juridique, à la psychiatrie transculturelle et à la psychologie interculturelle, au domaine des communications et à plusieurs professions de l'aide. Plusieurs propositions ont été faites par Goodale (2006a, 2006b) et ses collaborateurs, en particulier Engle Merry (Goodale et Merry, 2007), à propos de la direction que pourrait prendre une anthropologie des droits humains. Dépassant la vision portée par l'éternel débat relativisme/universalisme, Goodale et sa collègue Engle Merry en sont venus à dessiner les balises d'une anthropologie qui ne se limiterait ni au rôle de critique aveugle des droits humains considérés comme un produit de l'hégémonie occidentale et universaliste, ni au rôle de défenseur privilégié des cultures "uniques, fragiles et menacées", notamment par cette hégémonie supposée. Leur point de vue a plutôt permis de reconnaître le problème posé par cette hégémonie (réelle) du discours des droits humains et de ce qu'il véhicule sur le plan des moralités. Il a permis également de reconnaître la complexité qui se dessine aujourd'hui pour tout anthropologue désireux de développer des connaissances en lien avec les pratiques, les discours et les idéologies reliés aux droits humains, considérant le fait que certains groupes n'adhèrent pas à ce discours et à ses préceptes car ils refléteraient mal selon eux leurs ontologies, alors que d'autres, au contraire, s'en nourrissent en combinant le plus souvent les ontologies locales et les logiques universalistes des droits humains, en quelque sorte en les "vernacularisant », ou si l'on préfère, en les adaptant

\footnotetext{
${ }^{1}$ Dans ce numéro, l'expression "droits humains ", quoique discutable d'un point de vue purement linguistique, a été adoptée en référence au cadre anthropologique de la recherche. Elle a aussi l'avantage de mieux respecter les deux genres humains. L'expression "droits de l'homme " a été conservée en référence au contexte juridique de la Déclaration universelle des droits de l'homme » de l'ONU de 1948, et l'expression "droits de la personne » au contexte juridique canadien actuel. L'expression "droits humains » renvoie également plus explicitement à la traduction des « human rights » tels que les conçoit Mark Goodale, c'est-à-dire à une vision plus ouverte et globale que simplement légale. Voir Benhyeklef (2008) et Goodale (2006a).
} 
localement. Ainsi, ces auteurs, et de nombreux autres adoptant une position similaire (Asad, 2000; Engel, 2006; Hastrup, 2001a, 2001b et 2003; Mutua, 2002; Wilson, 2001 et 2006), proposent de s'appuyer sur des approches de tradition critique pour l'analyse des usages hégémoniques des droits comme celle des formes d'appropriations stratégiques ou circonstanciées auxquelles ils donnent lieu; ils proposent également de participer au travail d'élaboration et d'élargissement des cadres normatifs des droits (les textes), entre autres afin de rendre visibles les facteurs qui à ce jour ont limité l'accès aux droits collectifs ou ont rendu difficiles les imbrications harmonieuses entre droits et cultures. Il s'agirait de développer une approche certes critique des droits humains, mais sensible à leur caractère perfectible, et d'ouvrir à un travail collaboratif entre instances de gouvernance, populations, société civile, experts et gouvernants; il s'agirait également de mettre au point des méthodes innovantes ou plus adaptées. On ne pense pas ici à limiter le rôle de l'anthropologue à celui de traducteur des idiosyncrasies culturelles ou encore à celui de médiateur de l'idéologie des droits humains, mais d'ouvrir la voie à des approches moins normatives (entendre ici d'ordre strictement juridico-politique), moins dichotomiques (basées sur l'opposition universalisme-relativisme), cela afin de laisser place aux discours et aux pratiques des acteurs des droits humains, incluant les anthropologues eux-mêmes. Bref, de déborder du formalisme des textes et de leurs applications légalistes et d'entrer dans le vif des discours et des pratiques multisitués et qui ont cours dans diverses sociétés. L'anthropologie, par ses approches localisées, permet justement d'offrir un certain nombre de réponses en ramenant les pratiques liées aux droits au cœur de la vie sociale, là où par exemple ils se fabriquent ou se défont, sont appropriés ou transformés et réécrits. L'anthropologie, et avec elle d'autres corps disciplinaires, est susceptible de confronter le droit aux questions de pluralité et d'interculturalité qui traversent la vie sociale; elle nous rappelle à quel point les catégories du langage et de l'expérience ne sont pas si facilement réductibles à celles du droit.

C'est dans la continuité de ces débats que l'étude anthropologique des usages du témoignage à des fins de droits est rendue possible : sorti de l'espace juridique au sens étroit de la chose, par exemple le cadre du plaidoyer dans une cour, et observé puis analysé dans ses multiples manifestations sociales, notamment dans ses diverses articulations à l'univers des pratiques et discours sur les droits dans l'espace public et dans les sociétés contemporaines.

\section{Violence, témoignage et culture}

Ce numéro propose une série d'articles à caractère ethnographique et analytique sur les diverses utilisations du témoignage à des fins de droits dans plusieurs contextes et espaces sociaux et culturels. Une thématique particulière traverse l'ensemble du propos, celle de l'expérience, passée ou actuelle, de la violence subie par ceux qui, directement ou indirectement, ont recours au témoignage pour faire apparaître la problématique des droits qui les concernent. En effet, ce n'est pas de façon inopinée que les droits prennent caractère, relief et existence dans une société : des événements doivent avoir eu lieu, des droits et des humains doivent avoir été bafoués, des existences individuelles et collectives mises en cause. Il s'agit, à travers les différentes études proposées, de poser la question de l'usage du témoignage dans la vie sociale, dans des contextes culturels permettant d'en saisir la diversité, les limites et les enjeux. Le premier article, celui de Karoline Truchon, nous propose une histoire du témoignage dans le temps long des sociétés occidentales, puis dans leur histoire récente: comment les sociétés occidentales ont-elles pu avoir recours au témoignage comme forme de révélation et d'expression des manquements aux droits et des situations d'injustice? Comment la figure du témoin s'est-elle construite au fil du temps, et comment le témoignage prend-il, depuis le milieu du $x x^{\mathrm{e}}$ siècle, des formes de plus en plus diversifiées? Le deuxième article, celui de Martin Hébert, éclaire les réflexions de Truchon, en montrant que le témoignage s'inscrit d'abord et avant tout dans un récit collectif liant souffrance, culture et violence. Les cultures proposent en fait des modèles (patterns) de présentation et d'interprétation de la souffrance qui donnent au récit un caractère préconstruit, cela afin de le rendre recevable et lisible par le plus grand nombre. II en est ainsi des sociétés latinoaméricaines et des mondes autochtones étudiés par Hébert. L'expérience de la souffrance, incluant celle des manquements aux droits les plus fondamentaux, n'est jamais directement lisible, elle passe par des codes ou des idiomes culturels plus ou moins forts, tant par la médiation de l'énonciateur que par celle du récepteur. Le travail de Martin Hébert peut être directement repris dans le contexte du témoignage qui est, comme on l'a vu en introduction, une mise en mots de la souffrance collective lorsqu'il est question de manquements aux droits : le témoignage peut tout à fait être compris comme un récit de souffrance liant violence et droits. La recherche de 
Francine Saillant permet d'approfondir la même question mais à partir d'un terrain différent. Il s'agit dans ce cas de saisir comment les expériences de la souffrance, de la violence et des manquements aux droits dont sont témoins les ONG de développement et d'aide humanitaire transitent par des récits et des images qui sont travaillées par différents intermédiaires, dont les services de communication des ONG eux-mêmes. Le travail du témoignage contribue dans ce contexte à une forme de lissage des expériences originelles faites dans les sociétés où interviennent ces ONG internationales, et aussi à une forme de réduction, cela de manière à les rendre justement recevables et compréhensibles pour le grand public. On a voulu comprendre comment les ONG utilisent le témoignage pour illustrer les manquements aux droits et aussi faire, directement ou non, la promotion des droits. II est important de saisir que le témoignage direct, celui des premiers intéressés, passe rarement par leur propre parole mais plutôt par les intermédiaires que sont les intervenants, laissant toute la place aux images des sujets du témoignage qui sont, dans ce cas, les populations du Sud que l'on cherche à présenter et à faire connaître à travers l'action des ONG. II est clair que les valeurs des sociétés occidentales pèsent largement sur les choix des communicateurs; les enjeux d'interculturalité et les choix effectués sont ceux, nous sommes forcés de l'admettre, qui nous ramènent sur les terrains des sociétés occidentales, de leurs grands publics et de leurs gouvernants. C'est à cette condition que circulent les « images et les voix du Sud ». Dans les articles de Hébert et de Saillant, ce sont les récits collectifs que les sociétés créent et recréent à propos de la souffrance, leurs codes et leurs contraintes qui sont mis en jeux, analysés et exposés, soulignant du coup ce qui pèse sur ces récits et sur les témoignages, que les contraintes soient culturelles, structurelles ou institutionnelles. Les résultats, on l'avait compris, sont fort différents, puisque le premier (Hébert) montre l'exacerbation des images et des expériences et la seconde (Saillant) insiste plutôt sur leur euphémisation. Les intentions sont les mêmes: traduire au sein de la culture ou entre les cultures une souffrance qui prend racine dans des inégalités sociales inscrites dans la longue durée.

Quatre articles nous amènent dans des mondes où la souffrance collective et les exactions liées aux droits humains prennent d'autres significations par les contextes et les acteurs, sujets et institutions, qui les mettent en œuvre : il s'agit du contexte d'un génocide (Rwanda), d'une guerre (Congo et au-delà,) d'une dictature (Argentine) et du monde des réfugiés et demandeurs d'asile (Canada et au-delà). L'article sur le Rwanda que propose Annie Laliberté prend un sens particulier en ce qu'il amène à une analyse des actions des institutions internationales et locales visant la restauration démocratique dans le contexte du Rwanda de l'après génocide. Dans ce cas, ce sont les institutions de coopération médiatique (dits médias de paix) qui montrent les limites des pratiques journalistiques censées permettre l'apparition dans l'espace public des enjeux de justice une fois perpétré le plus horrible des crimes. Le journaliste étranger (canadien dans ce cas), se fait le témoin des témoins alors que le contexte politique tente de refouler au plus profond les crimes en reconstruisant à ses fins le récit du génocide, en proposant une lecture manichéenne des justes et des injustes et en obligeant le témoin journaliste à adopter ce schéma. Les limites qui pèsent sur le récit journalistique en tant que témoignage sont immenses car elles montrent justement la naïveté de la solution démocratique prescrite et du remède que serait le journalisme de paix en situation post génocidaire : comment témoigner quand le récit collectif prescrit et imposé par un gouvernent autoritaire rend impossible les lignes d'interprétation qui ne diviseraient pas le monde entre agresseurs et victimes, puis entre victimes adoptant la bonne version du récit du génocide et d'autres qui deviendraient, ne s'y conformant pas, des traîtres. Les institutions de coopération se frottent ici à la fragilité du modèle démocratique, dont les enjeux d'implantation sont justement de sortir de ce manichéisme, et aussi à de fortes barrières culturelles et politiques. La proposition de Sylvie Bodineau consiste pour sa part en une analyse des discours des intervenants dans le domaine de la protection de l'enfance situés dans diverses organisations de protection des enfants soldats. Cette proposition donne aux lecteurs un bon exemple de la problématique de la victime, soit celle du témoin direct, et de la difficulté rencontrée par les enfants, éternels "vulnérables ", à passer justement de victimes à sujets et, de surcroît, à sujets de droit. L'article d'Alice Verstraeten nous déplace vers les disparus de l'Argentine et vers celles qui ont été les inlassables témoins de ces derniers : les Mères de la place de Mai, qui ont dénoncé la disparition de leurs proches et qui furent traitées comme des "folles", car on niait justement les disparitions. Leur présence constante sur la fameuse place de Buenos Aires, encore aujourd'hui, le retour permanent à la mémoire de la disparition et aux liens brisés, à l'impunité et à la violence négationniste, l'invention d'une ritualité urbaine devant l'absence de reconnaissance des droits des victimes, sont exemplaires. Ici, la richesse du contexte du témoignage, multiple, narratif et performatif, son étendue dans le temps, sa circulation internationale, lui donne une force d'exemple sans pareil. Comme le dit l'auteure, le témoignage devient ici ce fil tendu entre le dicible et l'indicible, une pratique de négociation et de transmission qui prend une signification élargie et transculturelle. Enfin, l’article 
de Nathalie Ricard suggère que la violence prend de nombreuses formes, puisque de la scène des guerres et des dictatures on se transporte vers celle des cours de justice qui octroient le statut d'immigrant aux "illégaux " que sont les demandeurs d'asile au Canada. Dans cette contribution, ces illégaux sont ceux qui, pour cause d'orientation sexuelle, demandent asile au Canada, personnes dont la provenance est diversifiée, tout comme l'expérience de leur allosexualité (autres formes de sexualité que l'hétérosexualité). La chercheure montre parfaitement que, dans le contexte juridique et culturel des instances de l'immigration, les agents sont fortement imprégnés des schémas culturels qui les habitent au sujet de l'allosexualité, supposant que les allosexuels étrangers ont incorporé les codes attendus des allosexuels nord-américains. Ainsi, l'allosexuel du Sri Lanka devrait ressembler à l'allosexuel du Village gai de Montréal afin que son récit, qui normalement doit faire la preuve de la discrimination dans son pays, soit crédible. Or ce récit se trouve mis à mal parce que, justement, l'allosexuel ne semble pas avoir « le physique de l'emploi ». Et Nathalie Ricard de souligner, à juste titre, à quel point il est dificile de prouver l'intimité de l'allosexuel dans pareil contexte. On comprend donc que cette étude, qui s'ajoute à d'autres sur les contraintes du témoignage à des fins de droits dans les instances de l'immigration (Saillant et Truchon, 2008; Truchon et Saillant, 2009), enrichit d'un élément important un dossier encore trop peu connu. Nathalie Ricard souligne de plus la puissance des codes culturels transformés en préjugés et stéréotypes à propos des réfugiés, doublement discriminés et cumulant le fardeau de l'origine et de l'orientation sexuelle, sans oublier les facteurs raciaux et de genre qui entrent souvent en ligne de compte.

Bertrand Lebouché et Joseph Josy Lévy nous entraînent sur un tout autre chemin, celui du témoignage en situation de maladie, en l'occurence le VIH/sida, dont le caractère épidémique et collectif, en même temps que traumatique, ne peut que nous interpeller. Le $\mathrm{VIH} / \mathrm{sida}$, plus que toute autre maladie, a donné lieu à des formes de témoignages publics, pensons au projet du patchwork des noms (http://www.aidsquilt.org/) aux nouveaux rituels qui se sont élaborés devant le vide des églises occidentales de longue tradition (Lafontaine, 2008), ou encore à la quantité de romans autobiographiques et testimoniaux publiés depuis le début de l'épidémie (Lévy et Nouss, 1994). Dans l'article proposé, ce sont les récits de malades qui prennent le sens de témoignages, en particulier ceux de malades afro-américains. Des questions morales et éthiques se posent aux chercheurs comme dépositaires de ces récits. Le témoignage comporte toujours une dimension éthique, puisque ceux qui en sont les dépositaires et les médiateurs, qu'ils soient témoins ou témoins de témoins, ramènent sur la place publique, non seulement des " expériences » et des "récits ", mais également des histoires personnelles dont les dimensions collectives mettent au défi la société tout entière, ses orientations et ses valeurs. Le VIH/sida sert ici d'étalon pour prendre toute la mesure de l'éthique du témoignage, et de l'importance qu'il peut prendre pour contrer l'exclusion causée par certaines formes de maladie.

Deux articles viennent compléter ce numéro thématique. Celui de Nathalie Gordon nous rappelle que le témoignage dans l'espace public déborde des mots et se déploie aussi par d'autres formes, notamment celle de l'art. L'histoire du réfugié iranien et artiste dont l'histoire est relatée se présente, certes, comme une forme de démarche que nous qualifierions de performative, l'art et ses traces, ici picturales, permettant au témoin de dire un indicible et de transcender les formes conventionnelles de la narration. L'article de Michaël LaChance pose enfin le défi du témoignage collectif par l'art, dans un bidonville de Bogòta, en relatant l'expérience d'une collectivité expulsée de son lieu de vie. Cette communauté y retourne avec un groupe d'artistes afin d'occuper le lieu, qui se transforme en espace de mémoire et de réinvention symbolique de la communauté. LaChance questionne par ailleurs le sens même du fait de témoigner dans un contexte d'exclusion, de même que le rôle des intellectuels dans le recueil du témoignage.

Grâce à ces contributions, l'intention est de mieux comprendre le "travail du témoignage " dans différents contextes culturels. En effet, le témoignage, sorti des contextes de son expression juridique, mais quand même associé de près aux quêtes de droits de groupes minorisés ou minoritaires, de même qu'à certaines formes empruntées par l'exportation de projets démocratiques, ne peut qu'exiger une confrontation aux exigences de la culture. La culture, nous l'avons conçue aussi bien dans ses usages les plus courants (ex. : groupes ethnoculturels), qu'à travers d'autres usages (culture nationale, culture institutionnelle, culture juridique). II s'est agi de dénaturaliser l'usage du témoignage et de sa relation au juridique comme moyen et preuve, afin de prendre acte de l'ampleur des possibilités expressives qu'il contient lorsque cette dénaturalisation est rendue possible. Il s'est agi aussi de saisir son caractère relationnel et sa problématisation interculturelle: il n'y a pas de témoignage sans 
relation, puisque le témoignage se fait à quelqu'un et devant quelqu'un. Cette relation, lorsqu'elle implique des sujets ou des groupes appartenant à diverses cultures, suppose un travail de la culture, des cultures en cause, bref, une part d'interculturalité. L'interculturalité non pas prise au sens de simple rencontre, mais de tensions révélées par la confrontation obligée aux différences, au-delà du fait que les enjeux de ces confrontations soient imprégnés des bonnes intentions, celles que recèlent en principe les causes du droit et de la justice.

\section{Références bibliographiques}

Asad, T. (2000). What do human rights do ? An anthropological enquiry, Theory and Events, 4(4). Récupéré sur le site de diffusion Muse (accès restreint) à : http://muse.jhu.edu/login?auth=0\&type=summary\&url=/journals/theory_and_event/v004/4.4asad.html

Benyekhlef, K. (2008). Une possible histoire de la norme. Les normativités émergentes de la mondialisation. Montréal : Éditions Thémis.

Engle, M. (2006). Transnational human rights and local activism: mapping the middle. American Anthropologist, 108(1), 38-51.

Goodale, M. (2009). Human rights. An anthropological reader. Malden : Blackwell.

Goodale, M. et Merry, S. (2008). The practice of human rights. New York : Cambridge University Press.

Goodale, M. (2006a). Toward a critical anthropology of human rights. Current Anthropology, 47(3), 485-511.

Goodale, M. (2006b). Introduction to anthropology and human rights in a new key. American Anthropologist, 108(1), 9-83.

Hastrup, K. (2001a). Legal culture and human rights. Copenhagen : Kluwer.

Hastrup, K. (2001b). Human rights on common grounds. The quest for universality. Copenhagen : Kluwer.

Hastrup, K. (2003). Violence, suffering and human rights. Anthropological Theory, 3(3), 309-323.

Lafontaine, C. (2008). Réinventer la mort : rites et deuil privé. Dans C. Lafontaine, La société postmortelle, Seuil, 2008, p. 193-199. (voir le dossier correspondant en ligne : http://agora.qc.ca/thematiques/mort.nsf/Documents/Rites_funeraires-Deuil_prive_invention_de_nouveaux_rites_par_Celine_Lafontaine)

Lévy, J. et Nouss, A. (1994). Sida-Fiction. Lyon : Presses Universitaires de Lyon.

Mutua, M. (2003). Human rights. A political and cultural critique. Philadelphia : University of Philadelphia Press.

Rude-Antoine, E. et Chrétien-Vernicois, G. (2009). Anthropologies et droits. État des savoirs et orientations contemporaines. Paris : Éditions Dalloz.

Saillant, F., et Truchon, K. (2008). Être plus que Corps, Lien social et politiques, 59, 61-74.

Sheper-Hugues, N. et Bourgeois, P. (2004). Violence in war and peace. Malden : Blackwell.

Truchon, K. et F. Saillant (2009). Les médias comme tribunal d'appel? De I'utilisation du tribunal d'appel comme contre-pouvoir par des personnes demandant le refuge au Canada et leurs alliés. Bulletin Vivre Ensemble, 16(55), 21-25.

Wilson, R. (2001). Human rights and the politics of culture. PoLAR : Political and Legal Anthropology Review, 24(2), 126-136.

Wilson, R. (2006). Toward a critical anthropology of human rights. Current Anthropology, 47(3), 485-511. 